\section{CONTRIBUIÇĀO AO ESTUDO DAS VIAS BILIFERAS NO SUS SCROFA DOMESTICA. SISTEMATIZAÇÃO DO RAMUS PRINCIPALIS SINISTER}

\author{
ANTONIO ALBERTO D'ERRICO \\ Professor Livre Docente \\ IRVÊNIA LUIZA DE SANTIS PRADA \\ Professor Livre Docente \\ NILSON FERREIRA \\ Professor Assistente Doutor \\ ANTONIO FERNANDES FILHO \\ Professor Livre Docente
}

\section{INTRODUÇĀO E LITERATURA}

O sistema bilifero apresenta-se, ainda hoje, como vastíssimo campo de investigação, especialmente quando se busca conhecimento mais pormenorizado, no que tange às mais importantes vias de drenagem do fígado, no suino. $\mathrm{Vi}$ sando ampliar o estudo do sistema excretor hepático, nesta espécie, metodizamos agora as vias responsáveis pela formação do ramus principalis sinister.

Consultando as obras clássicas de Anatomia Veterinária não encontramos qualquer informação referente ao aludido tema; quanto aos trabalhos especializados passamos a descrever as informações ali contidas.

OTTAVIANI 4 (1933), realizando estudo radiográfico comparativo das vias bilíferas intra-hepáticas, relata-nos, que no suíno adulto, os territórios direito e esquerdo do fígado não podem ser divididos. Assim, as vias bilíferas mostram-se de forma espraiada e constituidas pelo grande ducto bilífero esquerdo, aonde chegam aferentes conspícuos, delgados e o ducto direito; este acha-se caracterizado, nesta espécie, como via delgada a representar simples afluente do ducto esquerdo, que recebe o ducto cístico e se continua no colédoco.

$O$ ducto esquerdo acolhe de três a cinco afluentes primários, pertencendo ao lobo acessório esquerdo o principal dos ramos ventrais, por sua vez já os mais importantes. Dos afluentes ventrais do ducto esquerdo, observam-se dois ou três deles relacionados ao lobo acessório direito e, outro, ao lobo quadrado, lembrando que este último pode ser tributário do ducto direito.

NETTELBLAD 3 (1954), pesquisando 56 fígados de diferentes mamíferos, entre os quais 1 (um) de suíno, examina o ramificado do ductus hepaticus. Após a injeção dos órgãos com massa destinada à obtenção de preparações por corrosão, o A. indica-nos que o ramus principalis sinister depois de oferecer o ramus dorsalis sinister, mostra-se em " $V$ " fornecendo o ramus dorsocaudalis sinister e o ramus ventrocranialis sinister.

JABLAN-PANTIC 2 (1963), observando as vias biliferas intra-hepáticas, em 58 animais domésticos, dos quais 16 suínos, após injeção dos fígados com substância radiopaca, através do ductus choledocus, seguida de fixação pela formalina a $4 \%$, oferece o seguinte relato:

a) o ramus principalis sinister, mais longo e calibroso, drena a bile do lobus sinister lateralis, lobus sinister medialis, lobus quadratus e, às vezes, também, da parte medial do lobus dexter medialis;

b) os ramos responsáveis pelo escoamento da bile do lobus sinister lateralis apresentam-se em número de dois ou três, tais sejam, o ventral - ramus ventralis lobi sinistri lateralis, o médio - ramus medius lobi sinistri lateralis e o dorsal - ramus dorsalis lobi sinistri lateralis, sendo que as vezes esse último ramo pode surgir diretamente do ramus principalis sinister ou até do ramus principalis dexter;

c) o ramus lobi sinistri medialis compõe, em alguns casos, tronco comum com o ramus lobi quadrati;

d) o ramus lobi quadrati representa um dos afluentes do ramus principalis sinister, mas em casos excepcionais pode desembocar no drenador do lobus sinister medialis ou no ductus cysticus.

BOULOGNE1 (1972) estuda a segmentação hepática em 185 suínos, destinando 50 delas à observação das vias biliferas. Com o auxílio da dissecção, corrosão e radiografias, oferece-nos resultados que, assim se sintetizam.

a) o canal biliar esquerdo representa o tronco dos ramos do lobo lateral esquerdo $(98,0 \%)$, do lobo medial es- 
querdo, lobo quadrado e, eventualmente $(6,0 \%)$ da parte supraportal do lobo caudato.

b) o número de coletores que afluem de cada lobo é variável, apresentado o lobo lateral esquerdo - um $(2,0 \%)$ ou três $(98,0 \%)$; lobo medial esquerdo - um $(58,0 \%)$, dois $(36,0 \%)$ ou três $(6,0 \%)$; lobo, quadrado - um $(56,0 \%)$ ou dois $(44,0 \%)$.

\section{MATERIAL E MÉTODO}

Nossas observaçōes baseiam-se no estudo de 30 conjuntos, compreendendo, cada um, fígado e trato duodenal, retirados em bloco, de suinos, de raças não definidas, machos, adultos, de diferentes e não conhecidas idades, recolhidos no Instituto Biológico do Estado de São Paulo.

Isolado o conjunto, abrimos o duodeno ao longo da borda livre e, exposta a mucosa, procediamos a canalização do ducto colédoco através da papila duodenal maior, procurando esvaziar, tanto quanto possivel, o sistema bilífero, mediante massagens e compressðes; injetamo-lo a seguir, com solução de "Neoprene latex 650", contendo celobar (Laboratório Maurício Villela) em suspensão a 50,0\% ou vinyl (bakelite vinyl Bland VMCH) *Os 20 primeiros órgãos, submetidos ao tratamento inicial, foram sucessivamente radiografados, fixados em formol a $10 \%$ e dissecados; as últimas 10 peças sofreram o processo de corrosão parcial, que consistia em emergir parcialmente a face visceral do figado, em solução de ácido sulfúrico a $10 \%$, enquanto o parênquima da face diafragmática era fixado com injeção de formol a $10 \%$.

Os territórios hepáticos, nesta espécie, exceção feita ao lobo medial direito e lobo quadrado, em parte fundidos, são bem demarcados por incisuras interlobares profundas. Para delimitá-los, idealizamos plano imaginário que, dispostos perpendicularmente às superfícies diafragmáticas e visceral do fígado, corta a fossa da vesícula biliar, percorrendo-o no sentido do maior eixo e a atingir a impressão da veia cava caudal. Desta forma no território hepático esquerdo encontramos o lobus sinister lateralis, lobus sinister medialis e na porção intermediária, importante para o relato de nossos resultados, o lobus quadratus e pars supra-portalis do lobus caudatus.

O lobus sinister lateralis foi dividido, para melhor visualizar a sistematização, em cinco porções desiguais, correspondentes aos territórios dorsal $(1 / 5)$, médio $(3 / 5)$, ventral $(1 / 5)$.

$\mathrm{Na}$ sistematização das vias biliferas levamos em conta, apenas, os territórios escoados e não o calibre dos ductos, pelo fato de que as injeçőes enchem melhor os de situação superficial.

Cumpre esclarecer que, havendo dado realce aos coletores conspicuos, não estendemos nossa atenção às raizes dos ramos principais; em contraposição, sempre assinalavamos as contribuições implicadas na combinação das vias de escoamento de territórios distintos, independente neste, do calibre, da procedência e destinação delas, anotando, ainda, quando representavam curtos troncos, o correspondente número de componentes.

Convencionamos orientar os relatos no sentido periferia-centro, indicando os ramos anatomicamente diferenciados dos restantes, pelas mencionadas caracteristicas, até a sua reunião com outros de igual calibre, para compor tronco inominado.

\footnotetext{
* Union Carbide Corporation - Chemical and Plastic. N.Y. USA
}

Adotamos na apresentação dos resultados; a nomenclatura empregada por JABLAN-PANTIC, identificando os ramus medius lobi sinistri lateralis, do focalo A., pelas denominações de ramus dorsomedialis lobi sinistri lateralis (porção dorsal do território médio) e do ramus ventromedialis lobi sinistri lateralis (porção ventral do território médio).

\section{RESULTADOS}

\section{Sistematização do ramus principalis sinister}

Nos 30 fígados, descobrimos a integrarem o sistema do ramus principalis sinister, o ramus dorsalis lobi sinistri lateralis, o ramus dorsomedialis lobi sinistri lateralis, o ramus ventromedialis lobi sinistri lateralis e o ramus ventralis lobi sinistri lateralis - constantes de todos os casos - além do ramus lobi sinistri medialis e do ramus lobi quadrati ambos sempre presentes, exceção feita a uma observação, do ramus lobi dextri medialis - 3 vezes $(10,0 \% \pm 5,5)$, do ductus cysticus -4 vezes $(13,3 \% \pm 6,2)$ e de contribuições inominadas, provenientes do lobus sinister lateralis (território dorsal, dorsomedial, ventromedial e ventral), lubus sinister medialis, lobus quadratus, lobus caudatus (pars supraportalis) e lobus dexter medialis.

1. O ramus dorsalis lobi sinistri lateralis e o ramus dorso-medialis lobi sinistri lateralis concorrem para tronco comum, em 24 das 30 peças examinadas $(80,0 \% \pm 7,3-$ Figs. 1 e 2). Os dois coletores urgem a escoarem somente as regiōes que lhes correspondem em 13 dos órgãos $(43,3 \% \pm$ $9,0)$ mas, recebem tributários -2 vezes $(6,7 \% \pm 4,6)$, isto é, ao primeiro deles vem ter um eferente do lobus sinister lateralis (território dorsomedial) - l vez $(3,3 \% \pm 3,3)$ e outro do lobus sinister medialis - 1 vez $(3,3 \% \pm 3,3)$ enquanto o segundo deles é alcançado por contingentes vindos do lobus sinister lateralis (território dorsal e dorsomedial), ou seja, apenas um, proveniente do território dorsal - I vez $(3,3 \% \pm 3,3)$ e igual contribuição ligada a dois coletores do território dorsomedial -1 vez $(3,3 \% \pm 3,3)$. Em 2 preparações $(6,7 \% \pm 4,6)$, vemos exclusivamente o ramus dorsalis lobi sinistri lateralis a acolher tributário, e procedente do lobus sinister lateralis (território dorsomedial). Quando somente o ramus dorsomedialis lobi sinistri lateralis recebe afluentes -7 vezes $(23,3 \% \pm 7,7)$ identificamo-los a procederem do lobus sinister lateralis território dorsal e ventromedial), a saber, um -4 vezes $(13,3 \% \pm 6,2)$, dois -1 vez $(3,3 \% \pm 3,3)$ ou três deles -1 vez $(3,3 \% \pm 3,3)$, nascidos do território dorsal, e outros dois, provenientes do território ventromedial -1 vez $(3,3 \% \pm 3,3)$.

A seu turno, o coletor comum ao ramus dorsalis lobi sinistri lateralis e ao ramus dorsomedialis lobi sinistri lateralis mostra-se livre em 5 das 24 focadas glândulas $(16,7 \% \pm$ $6,8)$. Entretanto, a eles chegam, nas mais $19(63,3 \% \pm 6,8)$, ductos originários:

a) exclusivamente do lobus sinister lateralis (territórios dorsal e dorsomedial), em 16 peças $(53,3 \% \pm 9,1)$ ou seja, um -7 veus $(23,3 \% \pm 7,7)$, dois -6 vezes $(20,0 \% \pm 7,3)$ ou três -1 vez $(3,3 \% \pm 3,3)$ coletores da primeira das regiőes apontadas, contribuição da segunda delas - $1 \mathrm{vez}$ $(3,3 \% \pm 3,3)$ e um contingente de cada procedencia, na ordem citada -1 vez $(3,3 \% \pm 3,3)$.

b) simultaneamente do lobus sinister lateralis (território dorsal) e lobus caudatus (pars supraportalis), isto é um afluente de cada origem - 1 vez $(3,3 \% \pm 3,3)$. 
c) concomitantemente do lobus sinister lateralis (território dorsal) e lobus sinister medialis, a saber, quatro eferentes do primeiro dos lobos, separados e seguidos de coletor do segundo deles -1 vez $(3,3 \% \pm 3,3)$.

d) simultaneamente do lobus caudatus (pars supraportalis) e lobus sinister medialis, vale dizer, duas contribuiçōes da pars supraportalis, independentes, e uma do lobus sinister medialis -1 vez $(3,3 \% \pm 3,3)$.

2. O ramus ventromedialis lobi sinistri lateralis e o ra mus ventralis lobi sinistri lateralis compõem tronco, em 24 dos 30 casos que analisamos $(80,0 \% \pm 7,3$ - Figs. 3 e 5$)$, sendo encontrados ambos livres -15 vezes $(50,0 \% \pm 9,1)$. Evidenciamos, em 1 caso $(3,3 \% \pm 3,3)$, o primeiro deles a receber afluentes vindos do lobus sinister lateralis, mais exatamente, um do território dorsomedial seguido de outro do território ventral, enquanto ao segundo, o ramus ventralis lobi sinistri lateralis vem ter, separadamente, duas contribuições do lobus sinister medialis. Ressaltamos que as mais 8 glândulas $(26,7 \% \pm 8,1)$ apresentam somente o ramus ventralis lobi dextri lateralis a escoar, apenas, o território que the corresponde, isto é, ao ramus ventromedialis lobi dextri lateralis destinam-se eferentes, e do lobus sinister lateralis (território ventral, dorsomedial e ventromedial); eles, em número de um, provém da primeira das regiões -3 vezes $(10,0 \% \pm 5,5) \mathrm{e}$, de dois, têm origem novamente na primeira - 1 vez $(3,3 \% \pm 3,3)$ ou na segunda e terceira, constituindo-se dupla de troncos iguais -1 vez $(3,3 \% \pm 3,3)$.

Por sua vez, a via comum ao ramus ventromedialis lobi sinistri lateralis e ramus ventralis lobi sinistri lateralis que identificamos nas citadas 24 preparaçðes, exibe-se livre de afluentes -15 vezes $(50,0 \% \pm 9,1)$; nos restantes 9 casos $(30,0 \% \pm 8,4)$, achamo-la a acolher tributários oriundos:

a) apenas do lobus sinister medial is 4 vezes $(13,3 \% \pm$ $6,2)$, contando-se um -3 vezes $(10,0 \% \pm 5,5)$ - ou dois deles -1 vez $(3,3 \% \pm 3,3)$;

b) exclusivamente do lobus sinister lateralis (territórios dorsomedial, vetromedial e ventral) -3 vezes $(10,0 \% \pm 5,5)$, isto é, um do primeiro -1 vez $(3,3 \% \pm 3,3)-$ um do segundo -1 vez $(3,3 \% \pm 3,3)$ e um do terceiro - 1 vez $(3,3 \%$ $\pm 3,3)$;

c) simultaneamente do lobus sinister medialis e lobus sinister lateralis (territórios dorsomedial e ventromedial) 2 vezes $(6,7 \% \pm 4,7)$, ou seja, duas contribuições, independentes, vindas da primeira e da segunda das regioes apontadas -1 vez $(3,3 \% \pm 3,3)$ e coletor da terceira delas associa. do a contingente nascido do lobus sinister medialis -1 vez $(3,3 \% \pm 3,3)$.

3. O ramus lobi sinistri medialis e o ramus lobi quadrati associam-se diretamente, em 10 dos 30 fígados (33,3\% t 8,6 - Fig. 1), aparecendo ambos livres -3 vezes $(10,0 \% \pm$ $5,5)$. Entretanto, descobrimos 4 dos órgãos $(13,3 \% \pm 6,2)$, a exibirem o ramus lobi quadrati recebendo um afluente vindo do lobus sinister medialis. De outra parte, apenas ao ramus lobi sinistri medialis chegam contribuiçōes originárias:

a) unicamente do lobus quadratus, em número de duas -1 vez $(3,3 \% \pm 3,3)$;

b) simultaneamente do lobus quadratus e do l lobus sinister medialis, ou seja, uma de cada procedência, unidas por tronco -1 vez $(3,3 \% \pm 3,3)$.

c) somente do lobus sinister medialis, isto é uma -1 vez $(3,3 \% \pm 3,3)$.

A seu turno, o ducto constituído pela convergência do ramus lobi sinistri medialis e ramus lobi quadrati surge livre -8 vezes $(26,7 \% \pm 8,1)$ e recebe tributários, em 2 casos $(6,7 \% \pm 4,6)$, a saber, um proveniente do lobus sinister medialis -1 vez $(3,3 \% \pm 3,3)$ e um do lobus quadratus $-1 \operatorname{vez}(3,3 \% \pm 3,3)$.

4. O ramus dorsomedialis lobi sinistri lateralis e o ramus ventromedialis lobi sinistri lateralis apresentam-se diretamente unidos em 5 dos 30 órgãos $(16,7 \% \pm 6,8)$ permanecendo, o primeiro deles, a drenar exclusivamente o próprio território. Ao ramus ventromedialis lobi sinistri lateralis endereça-se, entretanto, em 2 casos $(6,7 \% \pm 4,6)$, contribuição proveniente do lobus sinister lateralis (território ventral).

Quanto à via que liga os dois focados ramos, encontramo-la livre -4 vezes $(13,3 \% \pm 6,2)$ e a receber -1 vez $(3,3 \% \pm 3,3)$ eferente do lobus sinister lateralis (território vent romedial).

5. O ductus cysticus e o ramus dextri medialis são vistos, em 1 das 30 glândulas $(3,3 \% \pm 3,3)$, a convergirem para tronco comum, incorporado ao sistema do ramus principalis sinister.

Nesta peça, tanto o ductus cysticus quanto a via resultante exibem-se livres mas, o ramus lobi dextri medialis acolhe afluente vindo do lobus quadratus.

6. O ramus lobi quadrati e o ductus cysticus exibemse diretamente associados, em 1 dos 30 fígados $(3,3 \% \pm$ 3,3 ), e livres de tributários, assim como o tronco que os reune.

7. O rumus ventralis lobi sinistri lateralis e o ramus lobi sinistri medialis, em 1 das 30 glândulas $(3,3 \% \pm 3,3)$ surgem diretamente fundidos e livres de afluentes, o mesmo acontecendo com o ducto que lhes é comum.

8. O ramus dorsalis lobi sinistri lateralis liga-se diretamente ao ramus ventrolateralis lobi sinistri lateralis, em 1 das 30 peças $(3,3 \% \pm 3,3)$, quando o segundo deles aparece livre, assim como a via resultante, e o primeiro, isto é, o ramus dorsalis lobi sinistri lateralis acolhe dois contingentes vindos do lobus sinister lateralis (território dorsomedial).

Nos casos em que os ductos nominados não se ligam diretamente a outros coletores de igual categoria, vão ter a tronco que reune dois ou mais deles.

1. O ramus dorsalis lobi sinistri lateralis, já o dissemos, une-se ao ramus dorsomedialis lobi sinistri lateralis em 24 das 30 preparações $(80,0 \% \pm 7,3$ - Figs. 1 e 2$)$ e ao ramus ventromedialis lobi sinistri lateralis -1 vez $(3,3 \% \pm 3,3)$. Nas restantes 5 glândulas $(16,7 \% \pm 6,8)$, ele alcança ductos resultantes da união sucessiva:

a) do ramus dorsomedialis lobi sinistri lateralis mais ramus ventromedialis lobi sinistri lateralis, ramus ventralis lobi sinistri lateralis e ramus dorsalis lobi sinistri lateralis -3 vezes $(10,0 \% \pm 5,5)$. O ramus dorsalis lobi sinistri lateralis recebe, em todos os casos, eferentes do lobus sinister lateralis (território dorsomedial), isto é, um -2 vezes $(6,7 \% \pm 4,6)$ ou dois - 1 vez $(3,3 \% \pm 3,3)$, enquanto a via final, livre -2 vezes $(6,7 \% \pm 4,6)$ recebe -1 vez $(3,3 \% \pm$ $3,3)$, coletor oriundo do lobus sinister lateralis (território dorsal);

b) do ramus dorsomedialis lobi sinistri lateralis mais ramus ventromedialis lobi sinistri lateralis -1 vez $(3,3 \% \pm$ $3,3)$. Vemo-lo então a acolher três eferentes do lobus sinister lateralis (território dorsomedial) e, o ducto resultante, a receber contribuição vinda do lobus sinister medialis; 
c) do ramus dorsomedialis lobi sinistri lateralis mais ramus ventromedialis lobi sinistri lateralis, ramus ventralis lobi sinistri lateralis e ramus lobi sinistri medialis mais ramus lobi quadrati -1 vez $(3,3 \% \pm 3,3)$. Neste caso, ao ramus dorsalis lobi sinistri lateralis vem ter afluente nascido no lobus sinister lateralis (território ventral) seguindo de outro do lobus sinister medialis; à via final endereça-se um eferente do lobus sinister lateralis (território dorsal e dois coletores do lobus quadratus, o último deles associado a contribuição do lobus dexter medialis.

$2 \mathrm{O}$ ramus ventralis lobi sinistri lateralis, consoante afirmamos, associa-se, em 24 dos 30 órgãos $(80,0 \% \pm 7,3$ Figs. 1 e 2) ao ramus ventromedialis lobi sinistri lateralis e, 1 vez $(3,3 \% \pm 3,3)$ ao ramus lobi sinistri medialis. Nas outras 5 peças $(16,7 \% \pm 6,8)$, achamo-lo, todavia, endereçada a ducto resultante da união dos seguintes coletores:

a) ramus dorsomedial is lobi sinistri lateralis mais ramus ventromedialis lobi sinistri lateralis -4 vezes $(13,3 \% \pm 6,2)$. Enquanto o ramus ventralis lobi sinistri lateralis aparece a drenar exclusivamente o próprio território, a via final, também livre -3 vezes $(10,0 \% \pm 5,5)$ recebe -1 vez $(3,3 \% \pm$ $3,3)$ contribuição do lobus sinister medialis;

b) ramus dorsalis lobi sinistri lateralis mais ramus ventromedialis lobi sinistri lateralis e ramus dorsomedialis lobi sinistri lateralis -1 vez $(3,3 \% \pm 3,3)$. Aqui, o ramus ventralis lobi sinistri lateralis surge livre de afluentes, ao passo que o tronco resultante das unioes citadas acolhe dois eferentes do lobus sinister medialis intercalado por contingente vindo do lobus sinister lateralis (território dorsal).

3. O ramus lobi sinistri medialis, que apenas 1 vez $(3,3 \% \pm 3,3)$ não participa do sistema do ramus principalis sinister, conforme descrevemos, liga-se ao ramus lobi quadrati, em 10 dos 30 fígados $(33,3 \% \pm 8,6)$ e ao ramus ventralis lobi sinistri lateralis - 1 vez $(3,3 \% \pm 3,3)$. Encontramo-lo, nas mais $18(60,0 \% \pm 8,9)$, dirigido a ducto constituido pelos integrantes:

a) ramus ventromedialis lobi sinistri lateralis mais ramus ventralis lobi sinistri lateralis - 10 vezes $(33,3 \% \pm$ $8,6)$. Surpreendemos, aqui, o ramus lobi sinistri medialis $\mathrm{li}$ vre -9 vezes $(30,0 \% \pm 8,4)$ e a receber tributário oriundo do próprio território, associado a outro do lobus quadratus - 1 vez $(3,3 \% \pm 3,3)$. A seu turno, a via que reune os três ramos citados também surge livre -8 vezes $(26,7 \% \pm 8,1)$ acolhendo, nas mais $2(6,7 \% \pm 4,6)$, afluente vindo do lobus quadratus -1 vez $(3,3 \% \pm 3,3)$ e contribuição do lobus sinister medialis seguida de outra do lobus sinister lateralis (território dorsomedial) - 1 vez $(3,3 \% \pm 3,3)$.

b) ramus dorsalis lobi sinistri lateralis mais ramus dorsomedialis lobi sinistri lateralis e ramus ventromedialis lobi sinistri lateralis -6 vezes $(20,0 \% \pm 7,3)$, quando o ramus lobi sinistri medialis drena exclusivamente o próprio território, ao passo que o tronco final é livre -5 vezes $(16,7 \% \pm$ $6,8)$ e recebe -1 vez $(3,3 \% \pm 3,3)$, dois eferentes do lobus sinister lateralis, isto é, um do território dorsal e outro do dorsomedial, unidos por ducto comum;

c) ramus dorsomedialis lobi sinistri lateralis mais ramus ventromedialis lobi sinistri lateralis, ramus ventralis lobi sinistri lateralis e ramus dorsalis lobi sinistri lateralis -2 vezes $(6,7 \% \pm 4,6)$. Em ambos, o ramus lobi sinistri medialis aparece livre mas, a via resultante vem ter tributários, ou seja, contingente nascido no lobus sinister lateralis (território dorsal) seguido de outro, vindo do lobus sinister medialis ligado a contribuição do lobus quadratus - 1 vez $(3,3 \%$ $\pm 3,3)$ e quatro coletores independentes, oriundos, por ordem, do lobus sinister lateralis (território dorsal), lobus sinister medialis, lobus caudatus (pars supraportalis) e lobus quadratus -1 vez $(3,3 \% \pm 3,3)$.

4. O ramus lobi quadrati, já o afirmamos, somente em 1 dos 30 órgãos $(3,3 \% \pm 3,3)$ não integra o sistema do ramus principalis sinister; conjuga-se 10 vezes $(33,3 \% \pm 8,6$ Fig. 1), ao ramus lobi sinistri medialis e, 1 vez $(3,3 \% \pm$ $3,3)$, ao ductus cysticus. Nas outras 18 preparaçסes $(60,0 \%$ $\pm 8,9$ - Fig. 2), ele se lança nos ductos formados pela convergência dos ramos:

a) ramus dorsalis lobi sinister lateralis mais ramus dorsomedialis lobi sinistri lateralis, ramus ventromedialis lobi sinistri lateralis mais ramus ventralis lobi sinistri lateralis e ramus lobi sinistri medialis -14 vezes $(46,7 \% \pm 9,1)$. Nestes casos, o ramus lobi quadrati mostra-se livre - 11 vezes $(36,7 \% \pm 8,8)$ e acolhe, nas outras $3(10,0 \% \pm 5,5)$, contribuição do lobus sinister medialis. Por sua vez, o tronco final, já configurando o próprio ramus principalis sinister, em 11 casos $(36,7 \% \pm 8,8)$, também aparece livre 12 vezes $(40,0 \% \pm 8,9)$, recebendo coletor do lobus quadratus mais contribuição do lobus sinister lateralis (território dorsal) associada a outra do lobus caudatus (pars supraportalis) - 1 vez $(3,3 \% \pm 3,3)$ e eferente do lobus sinister lateralis (território dorsal) -1 vez $(3,3 \% \pm 3,3)$;

b) ramus ventromedialis lobi sinistri lateralis mais ramus ventralis lobi sinistri lateralis e ramus lobi sinistri medialis -2 vezes $(6,7 \% \pm 4,6)$, ocasioes em que o ramus lobi quadrati é livre -1 vez $(3,3 \% \pm 3,3)$ recebendo, na outra $(3,3 \% \pm 3,3)$, contingente vindo do lobus sinister medialis. Quanto à via que reune os focados ramos, surge livre de tributários, em ambos os casos;

c) ramus dorsomedialis lobi sinistri lateralis mais ramus ventromedialis lobi sinistri lateralis, ramus ventralis, lobi sinistri lateralis, ramus dorsalis lobi sinistri lateralis e ramus lobi sinistri medialis -1 vez $(3,3 \% \pm 3,3)$, quando o ramus lobi quadrati, assim como a via resultante, - já representativa do próprio ramus principalis sinister - , aparece livre de afluentes;

d) ramus dorsomedialis lobi sinistri lateralis mais ramus ventromedialis lobi sinistri lateralis, ramus dorsalis lobi sinistri lateralis e ramus ventralis lobi sinistri lateralis mais ramus lobi sinistri medialis -1 vez $(3,3 \% \pm 3,3)$, ocasião em que o ramus lobi quadrati é livre e o tronco final - já configurando o próprio ramus principalis sinister - recebe coletor vindo do lobus sinister medialis seguido de outro, do lobus caudatus ( pars supraportalis).

5. O ramus lobi dextri medialis, consoante relatamos, faz parte do sistema do ramus principalis sinister em 3 das 30 glândulas $(10,0 \% \pm 5,5), 1$ das quais $(3,3 \% \pm 3,3)$ mostra-o associado diretamente ao ductus cysticus. As outras 2 $(6,7 \% \pm 4,6)$ exibem-no endereçado a tronco dos seguintes coletores:

a) ramus dorsomedialis lobi sinistri lateralis mais ramus ventromedialis lobi sinistri lateralis, ramus ventralis lobi sinistri lateralis e ramus lobi sinistri medialis -1 vez $(3,3 \% \pm$ $3,3)$, quando o focado ramus lobi dextri medialis e o tronco final aparecem livres;

b) ramus dorsalis lobi sinistri lateralis mais ramus dorsomedialis lobi sinistri lateralis, ramus lobi sinistri medialis mais ramus lobi quadrati e ramus ventromedialis lobi sinistri lateralis mais ramus ventralis lobi sinistri lateralis $-1 \mathrm{vez}$ $(3,3 \% \pm 3,3)$. Enquanto o ramus lobi dextri medialis acolhe dois afluentes, separados, vindos do lobus caudatus 
(pars supraportalis), a via resultante é livre já configura o próprio ramus principalis sinister.

6. $\mathrm{O}$ ductus cysticus, tal como descrevemos, compõem o sistema do ramus principalis sinister em 4 dos 30 fígados $(13,3 \% \pm 6,2)$; encontramo-lo, 2 vezes $(6,7 \% \pm 4,6)$, associado diretamente a outro ducto nominado, ou seja, o ramus lobi quadrati -1 vez $(3,3 \% \pm 3,3)$ e o ramus lobi dextri medialis -1 vez $(3,3 \% \pm 3,3)$. Todavia, nos 2 casos restantes $(6,7 \% \pm 4,6)$, ele vai ter a tronco formado por ramus ventromedialis lobi sinistri lateralis mais ramus ventralis lobi sinistri lateralis, ramus lobi sinistri medialis, ramus lobi quadrati e ramus dorsalis lobi sinistri lateralis mais ramus dorsomedialis lobi sinistri lateralis, surgindo livre, assim como a via final, que já se constitui no próprio ramus principalis sinister.

\section{COMENTÁRIOS}

Como as obras clássicas de Anatomia Veterinária, nada tratam sobre o focado assunto, os nossos resultados serão comparados apenas aos dados encontrados em trabalhos análogos.

No atinente à sistematização do ramus principalis sinister, JABLAN-PANTIC ${ }^{2}$ e BOULOGNE ${ }^{1}$, como nós, encontram o focado ramo a escoar, de modo geral o lobus sinister lateralis, o lobus sinister medialis e o lobus quadratus. Outrossim, o primeiro dos AA. aduz que o ramus principalis sinister mostra-se longo e calibroso drenando, também, às vezes, a parte medial do lobus dexter medialis, fato que evidenciamos em $10,0 \%$ dos casos, enquanto BOULOGNE 1 considera que para tal ramo escoa, também, eventualmente $(6,0 \%)$, a porção supraportal do lobo caudato; em nossas preparaçōes, igualmente registramos a presença de contribuiçōes dessa região, e dirigidas ao sistema do ramus principalis sinister, em 14 das 30 preparações, conforme se depreende da observação de nossos esquemas. De nossa parte, e diferentemente do relatado por esses AA. assinalamos ain$\mathrm{da}$, a integrarem o focado sistema, o ductus cysticus $(13,3 \%)$ e contingentes inominados vindos do lobus sinister lateralis (territórios dorsal, dorsomedial, ventromedial e ventral), lobus sinister medialis, lobus quadratus e lobus dexter medialis. Particularmente os coletores que efetuam a drenagem dos lobos citados, e que confluem para constituir o ramus principalis sinister, vemos que JABLAN-PANTIC 2 apresenta-os em número de três, quando relativos ao lobus sinister lateralis, tais sejam o ramus dorsalis lobi sinistri lateralis, 0 ramus ventralis lobi sinistri e o ramus medius lobi sinisti: quanto aos dois primeiros ramos, encontramo-los e descrevemo-los, inclusive sob a mesma terminologia mas, em correspondência ao ramus medius lobi sinistri, surpreendemos dois coletores, aos quais designamos de ramus dorsomedialis lobi sinistri lateralis e ramus ventromedialis lobi sinistri lateralis, dispostos sob diferentes arranjos em relação aos outros contingente integrantes do sistema do ramus principalis sinister. A seu tumo, BOULOGNEl escreve ter evidenciado, a drenarem o lobo lateral esquerdo, um $(2,0 \%)$ ou três $(98,0 \%)$ ductos. Por outro lado, segundo OTTAVLANI 4 , o ducto esquerdo acolhe de três a cinco afluentes primários, enquanto NETTELBLAD ${ }^{3}$ assinala que o ramus principalis sinister resulta da confluência de três contingentes, ou seja, o ramus dorsalis sinister, o ramus dorsocaudalis sinister e o ramus ventrocranialis sinister. Entendemos que a diversidade quanto ao número de ductos escoadores do lobo direito, registrada entre os vários $\mathrm{AA}$., talvez esteja ligada ao fato dos vários contingentes apresentarem-se sob diferentes com- binaçōes, e formando troncos ora mais, ora menos calibrosos. De nossa parte, o calibre dos ramos ou das vias que eles constituem, conforme já esclarecemos, não foi tido como característica mais importante na caracterização dos coletores, posto que consideramos o fato da injeção de massa distender preferentemente os de situação superficial; por tal razão, levamos em conta, também, as dimensőes do respectivo setor de escoamento, na identificação dos ramos, lembrando ainda que conseguimos estabelecer, durante o preparo das peças, correspondência entre eles e definidos territórios de drenagem, no lobus sinister lateralis.

\section{CONCLUSÕES}

Baseados no estudo das vias bilíferas pertinentes ao sistema do ramus principalis sinister, em 30 suinos, de raça ñ̃o definida, adultos, de diferentes e não conhecidas idades, chegamos às seguintes conclusões:

a) integram o sistem do ramus principalis sinister, o ramus dorsalis lobi sinistri lateralis, o ramus dorsomedialis lobi sinistri lateralis, o ramus ventromedialis lobi sinistri lateralis e o ramus ventralis lobi sinistri lateralis - constantes de todos os casos -, além do ramus lobi sinistri medialis e do ramus lobi quadrati $(96,7 \%)$, do ramus lobi dextri medialis (10,0\%), do ductus cysticus $(13,3 \%)$ e de contribuições inominadas provenientes do lobus sinister lateralis (territórios dorsal, dorsomedial, ventromedial e ventral), lobus sinister medialis, lobus quadratus, lobus caudatus (pars supraportalis) e lobus dexter medialis.

b) Uma vez individualizado, o ramus principalis sinister surge livre de afluentes, em 19 das 30 preparações $(63,3 \%)$ a ele chegando, entretanto, nas mais $11(36,7 \%)$, coletores procedentes: apenas do lobus caudatus (pars supraportalis) - $13,3 \%$ ), simultaneamente do lobus sinister medialis e lobus caudatus (pars supraportalis - 6,7\%); concomitantemente do Jobus quadratus e lobus caudatus (pars supraportalis) - $(3,3 \%)$; somente do lobus sinister lateralis $(3,3 \%)$; simultaneamente do lobus quadratus, lobus sinister lateralis (território dorsal) e lobus caudatus (pars supraportalis) $(3,3 \%)$; apenas do lobus quadratus $(3,3 \%)$; concomitantemente do lobus quadratus e lobus dexter medialis $(3,3 \%)$.

c) $\mathrm{O}$ ramus dorsalis lobi sinistri lateralis, o ramus dorsomedialis lobi sinistri lateralis, o ramus ventromedialis lobi sinistri lateralis, o ramus ventralis lobi sinistri lateralis, o ramus lobi sinistri medialis e o ramus lobi quadrati escoam, na condição de coletores principais, por ordem, os territórios dorsal, ventromedial e ventral do lobus sinister lateralis, o lobus sinister medialis e o lobus quadratus; neles ou nas vias que constituem, desembocam inúmeras contribuições inominadas provenientes das aludidas regiōes hepáticas e, ainda, do lobus caudatus ( pars supraportalis). 
D'ERRICO, A.A.; SANTIS PRADA, I.L.; FERREIRA, N.;

FERNANDES FILHO, A. Contribution to the study of the intra-hepatic bili ducts in swines. Sistematization of the ramus principalis sinister. Rev. Fac. Med. vet. Zootec. Univ. S. Paulo, $17(1 / 2): 45-50,1980$.

SUMMARY: It was sistematized, by dissection, corrosion cast and radio logical examination, the biliary ducts of 30 swines. The results of this investigation indicated:a) the system of the ramus principalis sinister is integrated by the ramus dorsalis lobi sinistri lateralis, ramus dorsomed ialis lobi sinistri lateral is, ramus ventromedialis lobi sinistri lateralis, ramus ventralis lobi sinistri lateralis, ramus lobi sinistri medialis, ramus lobi quadrati, ramus lobi dextri medialis and ductus cysticus. b) that system is also composed by inominate colectors from the lobus sinister lateralis, lobus sinister medialis, lobus quadratus lobus caudatus (pars supraportalis) and lobus dexter medialis.

UNITERMS: Anatomy, swines *; Biliary ducts*; Liver *

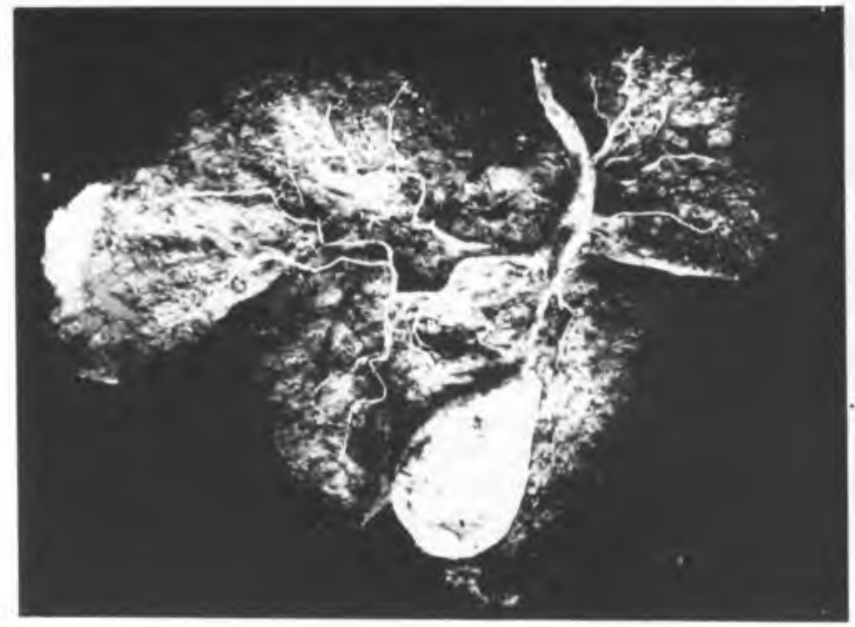

Figura 2

Fígado de suíno maclıo, adulto (Obs. 24), mostrando as vias bilíferas intra e extra-hepáticas preparadas segundo a técnica de injeção de "vinyl" (bakelite vinyl - Bland VMCH) seguida de corrquĩo controlada. Ao sistema do ramus principalis sinister (S) concorrem o ramus dorsalis lobi sinistri lateralis (L), o ramus dorsomed ialis lobi sinistri lateralis $(M)$, o ramus ventromedialis lobi sinistri lateralis $(N)$, $\circ$ ramus ventralis lobi lateralis $(O)$, o ramus lobi sinistri medialis $(P)$ e o ramus lobi quadrati $(\mathrm{R})$

\section{REFERÊNCIAS BIBLIOGRÁFICAS}

1 - BOULOGNE, J. C. La segmentation hepatique chez le porc domestique (Sus scrofa domesticus) Paris, 1972 (Thése - École Nationale Veterinaire D'Alfort).

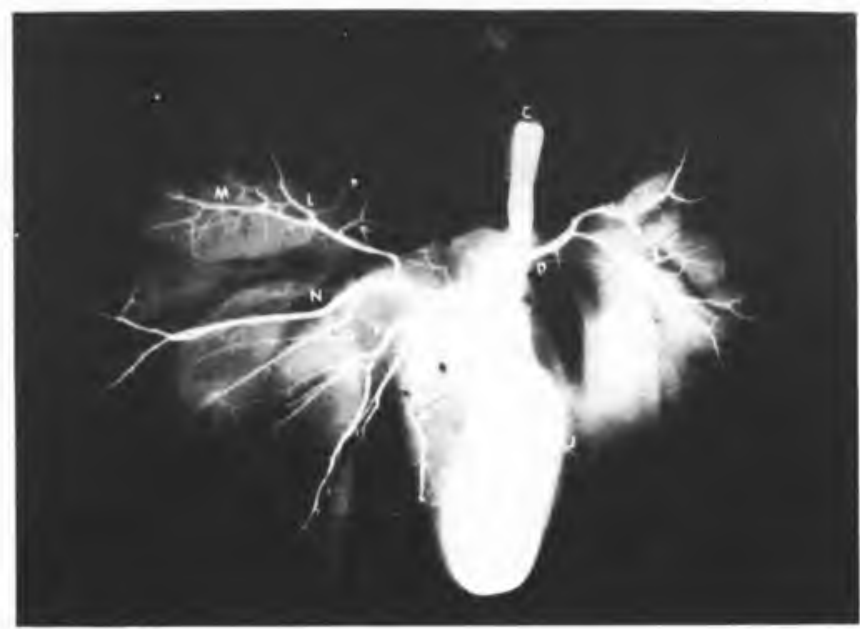

Figura 1

Fígado de suíno macho, adulto (Obs. 15); radiografia (incidência visceral) mostrando as vias bilíferas intra e extra-hepáticas injetadas com "Celobar" adicionando a "Neoprene látex 650 " $(50,0 \%)$. O ramus principalis dexter (D) e o ramus principalis sinister (S) confluem para formar o ductus hepaticus $(\mathrm{H})$ que, unido ao ductus cysticus (I), constitui o ductus choledocus (C). Nos fígados descobrimos a integrarem o sistema do ramus principalis sinister (S), como ductos principalis, o ramus dorsalis lobi sinistri lateralis (L), o ramus dorsomedialis lobi sinistri lateralis $(\mathrm{M})$, o ramus ventromedialis lobi sinistri lateralis $(N)$ e o ramus ventralis lobi sinistri lateralis $(O)$ constantes de todas as peças além do ramus lobi sinistri medialis ( $\mathrm{P}$ $(96,7 \%)$, do ramus lobi quadrati ( $R-96,7 \%)$, do ductus cysticus ( $\mathrm{I}$ $13,3 \%)$ e do ramus lobi dextri medialis $(\mathrm{J}-10,0 \%)$.
Recebido para publicação em: 18/08/1980 Aprovado para publicação em: 20/10/1980
2 - JABLAN-PANTIC, O. Caracteristics and comparative ratios of intra-hepatic bile duct in domestic animals. Acta vet., Belgrado, 13:3-14, 1963.

3 - NETTELBLAD, S. C. Die lobierung und innere topographie der Saugerleber nebst beitragen zur kenntnis der leberentwichlung beim goldhamster. Acta anat., Basil (supl. 20):1-251, 1954.

4 -- OTTAVIANI, G. Richerche radiografiche comparative sulle vie bilifere intraepatiche. Atti Inst. Veneto Sci., 92 (pt. 2) : 1096-8, 1933. 